\title{
Luchas por la escolarización de jóvenes en condición de pobreza de Argentina
}

\author{
Struggles for the schooling of young people \\ in poverty in Argentina
}

\author{
Eduardo Langer ${ }^{1}$ \\ langereduardo@gmail.com
}

\begin{abstract}
Resumen
Se trata de estudiar las dinámicas cotidianas de los dispositivos pedagógicos de manera comparativa en dos localidades de diferentes provincias de Argentina, Santa Cruz y Buenos Aires, atendiendo a las condiciones de vida, a las defensas y formas para sostener la educación de jóvenes en barrios en los que se fue profundizando la pobreza urbana y las desigualdades sociales de las comunidades. La preocupación es entender cómo las formas que adquieren las condiciones y las situaciones de vida de los sujetos se expresan, actúan e intervienen en la cotidianeidad escolar. Las dimensiones centrales de trabajo y comparativas - pobreza, escolarización y luchas sociales- se desarrollan en función de diferentes técnicas de obtención de información, a partir de una encuesta realizada en ambas provincias y de entrevistas en profundidad en ambos contextos.
\end{abstract}

Palabras clave ${ }^{2}$

Luchas, escolarización, dispositivos, comunidades, pobreza.

\begin{abstract}
It is studying the daily dynamics of pedagogical devices comparatively in two locations in different provinces of Argentina, Santa Cruz and Buenos Aires, considering the living conditions, defenses and ways to support youth education in neighborhoods where it was deepening urban poverty and social inequalities in communities. The concern is to understand how the ways they acquire the conditions and situations of life of the subjects are expressed, act and intervene in daily school life. The central dimensions of work and comparatives -poverty, schooling and social struggles-are developed based on different techniques for obtaining information from a survey conducted in both provinces and in-depth interviews in both contexts.
\end{abstract}

\section{Keywords}

Social struggles, schooling, pedagogical devices, communities, poverty.

Forma sugerida de citar: Langer, Eduardo (2016). Luchas por la escolarización de jóvenes en condición de pobreza de Argentina. Univeristas, XIV(1), pp. 119-142.

1 Doctor de la Universidad de Buenos Aires con mención en Educación. Profesor de Sociología de la Educación por la UNSAM y la UNPA.

2 Palabras claves extraídas del Tesauro Europeo de la Educación. 


\section{Introducción}

En Argentina hubo profundas transformaciones hacia los años noventa a partir de reformas políticas, económicas y educativas. Esos cambios de orientación conllevaron un aumento histórico en los índices de desocupación y empobrecimiento de amplios sectores sociales. Algunas de las consecuencias que se observan durante la primera década del siglo XXI, es que la población comenzó a reaccionar a partir de acciones organizadas en conflictos sociales así como a través de prácticas cotidianas en sus comunidades barriales como en las instituciones de las que son partícipes para la mejora de sus derechos sociales.

Aquí, se procura comprender esas reacciones cotidianas como luchas sociales por los derechos, específicamente por la escolarización, atendiendo a las prácticas y a las acciones que se realizan en contextos de pobreza. Se consideran esas acciones como luchas por sobrevivir, por tener algún lugar donde vivir, por trabajos dignos en la sociedad de empresa (Foucault, 2007), acciones que involucran tanto la resistencia hacia las consecuencias del capitalismo como a las formas de disciplinamiento y control. En este marco se trata de estudiar de manera comparativa las dinámicas cotidianas de los dispositivos pedagógicos atendiendo a prácticas de los sujetos en dos localidades en las que la pobreza urbana de las comunidades fue profundizándose, tanto dentro como fuera de las escuelas. Nos situamos en la preocupación por entender cómo las formas que adquieren las condiciones y las situaciones de vida de los sujetos se expresan, actúan e intervienen en la cotidianeidad escolar.

Se trata aquí de contribuir a los estudios sobre esas luchas que los jóvenes manifiestan cotidianamente: por sus identidades (Reguillo, 2012) frente al exceso de ira que producen formas nunca vistas de degradación y de incertidumbres (Appadurai, 2007); respondiendo al silencio, sufrimiento y sumisión de los olvidados y condenados junto con la humillaciones del sistema (Onfray, 2011); constituyéndose como nuevas clases de indignación (Hessel, 2006) fundamentalmente en los sectores de pobreza estructural a raíz de los procesos de crisis y de precariedad laboral; que conllevan acciones constructivas de los sujetos para denunciar y actuar ante un mundo injusto produciendo "ciudadanías insurrectas" (Holston, 2009, p. 57); como postulaciones de un orden distinto y, fundamentalmente, como dice Kristeva (1999) "contra la degradación del hombre” (p. 232); en definitiva, como formas de lucha que 
muestran "otros valores y comportamientos que se ponen en juego en las estrategias colectivas de subsistencia" (Redondo, 2004, p. 55).

En este artículo haremos foco y caracterizaremos esas luchas sociales desde tres dimensiones centrales de comparación: las situaciones y/o condiciones de pobreza de la población en ambos territorios, las ideas y valoraciones sobre la escolarización de los jóvenes a diferencia de sus docentes y las prácticas que realizan las familias de los jóvenes para defender y apostar por la educación.

Ello se realiza en función de múltiples técnicas de obtención de información, tanto cuantitativas como cualitativas. En cuanto a la primera técnica, se realizaron encuestas a estudiantes, docentes y padres en la Provincia de Santa Cruz y en el Partido de San Martín en 2008 y 2011, respectivamente 3. Los aspectos que se abordaron en las encuestas fueron sobre información sociodemográfica, trayectoria laboral y educativa, percepciones acerca del barrio de la escuela, consumos culturales, ideas sobre la escuela, régimen disciplinario en la escuela, ideas acerca del futuro (Langer, Venturini y Grinberg, 2013). En cuanto a la segunda técnica, se realizaron entrevistas en profundidad en el marco de proyectos de investigación de la Universidad Nacional de la Patagonia Austral y de la Universidad Nacional de San Martín durante 2014 y 2015. Allí buscamos conocer las situaciones y condiciones de vida de los jóvenes desde sus perspectivas tanto como la de sus familias -fundamentalmente, la de sus madres y padres. Los ejes de las entrevistas tuvieron que ver con las maneras de hacer, sentir y hablar de jóvenes y de las familias en condición de pobreza sobre sus formas de vivir, su educación, sus escuelas, sus luchas, estrategias, apuestas y defensas desplegadas para sostener la escolaridad cotidianamente, sobre sus deseos en relación a las posibilidades de seguir estudiando en función de sus condiciones de vida.

El proceso de análisis e interpretación de la información realizada a partir de la combinación metodológica nos permite el desarrollo de categorías conceptuales que se relacionan con los conceptos trabajados teóricamente.

3 Las encuestas realizadas en la Provincia de Santa Cruz durante 2008 fueron un total 2790 estudiantes, 117 docentes y 538 padres de los estudiantes en 25 escuelas públicas. Las encuestas realizadas en el Partido de San Martín durante 2011 fueron en un total de 15 escuelas públicas a 1179 estudiantes, 63 docentes y 116 a padres de estudiantes. Los criterios de selección de las escuelas fueron tipos de localizaciones geográficas de las instituciones atendiendo a los grados de urbanización, a la segmentación social de los espacios y las distintas zonas geopolíticas de las provincias; matrícula por escuela, resultados escolares tales como tasa de repitencia, abandono, deserción, promoción y no promoción de las escuelas (Langer, Venturini y Grinberg, 2013). 
En este sentido, la tarea primordial al analizar los discursos que siguen, retomando a Foucault (2007a), no es interpretarlo ni tampoco determinar si es veraz, sino trabajarlo desde el interior y elaborarlo, distinguir lo que es pertinente de lo que no lo es, definir unidades y describir relaciones porque son siempre "efectos de una construcción cuyas reglas se trata de conocer y cuyas justificaciones hay que controlar (p. 39). Se intenta captar esos enunciados en la singularidad de su acontecer, determinando las condiciones de su existencia a la vez que encontrar la intención del sujeto parlante y lo que ha querido decir en su contexto. Mediante la triangulación de la información que nos provee las encuestas semiestructuradas y las entrevistas individuales en profundidad, tratamos de mostrar en este trabajo un relato más completo de lo que cada una de ellas podría lograr sola. Así, a continuación comenzamos con la descripción de las situaciones y condiciones de vida de los jóvenes a los que nos referimos a lo largo del escrito.

\section{Las situaciones y condiciones de vida de los jóvenes estudiantes}

En Argentina, la crisis del fordismo y el proceso de desindustrialización que se inicia en los años 70 deterioraron y tuvieron un impacto clave tanto en el conurbano de Buenos Aires como en la zona norte de la Patagonia Austral donde se encontraban concentradas las industrias.

Tomando un lugar clave y central de Buenos Aires, el partido de General San Martín fue, entre otros, uno de los más afectados debido al cierre masivo de industrias de cristalería, automóvil y/o textil que transformó las viejas comunas obreras en verdaderos cementerios industriales (Prévôt Schapira, 2002). También, fue afectada la ciudad de Caleta Olivia frente a los procesos de privatización de una de sus principales actividades laborales que es la producción petrolera (Pérez, 2012). Estos procesos de crisis impactaron en los espacios generando una mayor polarización residencial de las áreas.

En el caso del Partido de San Martín, el surgimiento de nuevos asentamientos y villas da cuenta de una tendencia creciente del fenómeno de ocupación de tierras o de informalidad urbana, donde la precariedad de la tenencia de la vivienda es la norma que impera desde las últimas décadas del siglo XX. La localización de esta población está relacionada con la estratificación social, "los estratos bajos se localizan en áreas precarias de infraestructura y 
con problemas ambientales" (Shammah, 2009, p. 87). Así como el municipio es llamado "la Ciudad de la Tradición y Capital de la Industria", también se lo denomina la "Patria del Cirujeo"4 (Shammah, 2009, p. 95). El proceso de ocupación de la zona se corresponde con el devenir de la vida económica del país y en el Partido de San Martín se observa con especial crudeza ya que nos referimos a uno de los municipios que "hasta fines de los años setenta, era epicentro industrial del conurbano bonaerense, donde todavía, hoy, al entrar se puede ver un gran cartel que dice Bienvenido a la Capital de industria" (Grinberg, 2006, p. 5). Es decir, la ocupación de esta zona se produce en un movimiento paralelo que involucra el crecimiento y llegada de cada vez más gente a vivir a la zona, por un lado, y el cierre de las fábricas, por el otro.

En el caso de Caleta Olivia, la construcción del espacio urbano de la ciudad tuvo un quiebre a partir de 1990 hasta la actualidad a través de un tipo de capitalismo flexible (Harvey, 2012) en el que la privatización de los espacios de la estatal $\mathrm{YPF}^{5}$, la aparición o emergencia de conflictos por parte de sectores excluidos de los nuevos patrones económicos a partir del proceso de ajuste estructural de reforma neoliberal y "la llegada de flujos poblaciones diferentes a los patrones establecidos en los flujos denominados de redes de contacto hacia mediados del año 2000" (Pérez, 2012, p. 300).

Aquí encontramos la primera similitud en el patrón comparativo de la caracterización de estos contextos de pobreza: la intensidad de los flujos poblacionales que arriban tanto a Caleta Olivia como al partido de San Martín. Esa intensidad de los flujos poblacionales desborda la capacidad de servicios de Caleta Olivia (Pérez, 2012) y la de los barrios periféricos de San Martín, fundamentalmente las villas y los asentamientos.

Los barrios en los que se desarrolla la presente investigación crecieron al calor de los procesos de pauperización de la población, sistemáticos desde 1970 , y que se han visto atravesadas por los procesos de producción permanente de la crisis (Grinberg y Langer, 2012). Se trata de áreas donde cotidianamente se pone en cuestión el derecho a la ciudad (Lefebvre, 1978), y/o se constituyen en zonas de no derecho (Wacquant, 2007). Ello porque faltan o se accede de modo muy precario a los servicios cloacales, de agua potable,

4 El cirujeo o cirujas son la población marginal que vive de la basura recolectando cartón, papel y otros recursos que encuentran allí.

5 Empresa argentina dedicada a la exploración, explotación, destilación, distribución y venta de petróleo y sus productos derivados. 
de luz eléctrica, de recolección de basura sumado a la absoluta precariedad de las viviendas, del entramado de calles, así como a los establecimientos educativos, zonas de esparcimiento, espacios verdes, entre otras tantas características. Son barrios que combinan altos niveles de pobreza con altos niveles de contaminación ambiental, es decir, presentan todos los elementos típicos de un área hiperdegradada.

Para ejemplificar a través de nuestro trabajo de campo, la Tabla $\mathrm{N}^{\mathrm{o}} 1$ describe a jóvenes estudiantes que a la vez que estudian, trabajan; jóvenes con muchos hogares compuestos y sostenidos por beneficiaros de la ayuda social; y jóvenes con padres o madres que no poseen la escolaridad mínima.

\section{Tabla $\mathbf{N}^{0} 1$ \\ Algunas de las características de los jóvenes estudiantes de Caleta Olivia y de San Martín. En \%}

\begin{tabular}{|l|c|c|}
\hline & Caleta Olivia (N= 2790) & San Martín (N= 1179) \\
\hline Estudiantes que trabajan & 10,20 & 18,1 \\
\hline $\begin{array}{l}\text { Algún miembro del hogar } \\
\text { es beneficiario de un plan }\end{array}$ & 11,30 & 35,5 \\
\hline $\begin{array}{l}\text { Primario Incompleto de } \\
\text { padres y madres }\end{array}$ & 6,20 & 19,93 \\
\hline
\end{tabular}

Fuente y elaboración: Autor.

La Tabla $\mathrm{N}^{\mathrm{o}} 1$ expresa que los estudiantes de San Martín son quienes más trabajan (18.1\%) comparativamente con los de Caleta Olivia (10.2\%), aunque son en ambos lugares una proporción grande de jóvenes estudiantes que tienen algún empleo. También, nos hemos encontrado con que las familias de los estudiantes en estos barrios de ambas áreas han vivido y viven en gran medida por los subsidios o la ayuda social del Estado, trabajos ocasionales en el sector informal y las redes de intercambio social (Segura, 2006). Por último, una gran parte de las familias de los jóvenes no poseen la mínima educación, con mucho más fuerza en San Martín que la que se presenta en Caleta Olivia. Un supuesto de esta diferenciación es que los flujos poblacionales de ambas áreas tienen características distintas en cuanto a su composición educativa y en cuanto a sus objetivos laborales. Pero tanto en Caleta Olivia como en San Martín, la población migra en búsqueda de mejores oportunidades laborales, de vivienda, alimentarias y, por supuesto, también, de educación. 
Entonces, los contextos de pobreza a los que aquí nos referimos son barrios periféricos de Caleta Olivia y de San Martín en donde son caracterizados de diversas maneras por los sujetos: desde la desposesión material, la falta de infraestructura hasta el aumento de la violencia-fundamentalmente de la policía- y de la drogadicción. Son territorios de desposesión pero, también, de esperanza (Davis, 2007), de afirmación y de potencialidad. Son espacios donde la población oscila entre la búsqueda de prebendas del Estado (Zibechi, 2008), la rebelión y la dependencia de caudillos.

Si bien hay grandes diferencias y saltos entre las formas y las condiciones de vida en ambos lugares, también es cierto que se asemejan porque se constituyen comunidades que resisten contra el desplazamiento, el despojo y la incesante violencia cotidiana de la abyecta pobreza (Silberman-Keller et al., 2011; Grinberg, 2008) en las periferias urbanas. Las nociones de espacio urbano y luchas no son dos dimensiones separadas en tanto que los conflictos sociales están territorializados. Es en los barrios periféricos donde los sujetos insinúan e instituyen sus prácticas cotidianas, donde resisten y sobreviven, donde despliegan sus deseos y sus intereses, sus formas de ser, sus potencialidades.

De hecho, la noción de comunidad se constituye central para caracterizar la nueva arena sobre las cuales se asientan los planes y acciones de gobierno. Asistimos al gobierno a través de la comunidad (Rose, 1996a). Es decir, el de "comunidad" es una noción que expresa un registro de la asociación de cómo se reactiva la "pasión comunitaria de la participación en la vida pública" (Fistetti, 2004, p. 159) mediante la práctica concreta de la ciudadanía. Los sujetos tanto en las comunidades como en las escuelas en contextos de pobreza urbana de ambas áreas, adquieren responsabilidad para gestionarse a sí mismos con los recursos escasos con los que cuentan para sostener sus vidas y sus procesos de escolaridad en particular. Estas comunidades "son convocadas a participar en el gobierno de su propia seguridad" (De Marinis, 2002, p. 331), es decir, son individuos, agrupamientos, familias, movimientos, que construyen sus identidades y organizan sus opciones vitales manifestando un renovado énfasis sobre los contextos de la experiencia a través de diversidad de prácticas para articular sus demandas a autoridades de diverso tipo. El lenguaje de comunidad y de identidad se convierte en el sitio de nuevas contestaciones (Rose, 1996). Para Foucault (2006), justamente, la formación de comunidades es una de las maneras de mostrarse insumiso frente al poder porque es allí que se encuentran muchas otras ma- 
neras de destacar los esquemas de obediencia. En las comunidades hay "un aspecto de contrasociedad, de inversión de las relaciones y la jerarquía social, todo un lado de carnaval" (Foucault, 2006, p. 256).

De esta manera, los jóvenes a los que nos referimos se constituyen como participantes activos en sus vidas. De hecho, a partir de los datos provenientes de las entrevistas podemos visualizar en sus acciones, y también en las de sus familias y de sus docentes, los modos en que procuran moldear e intervenir, a través de las elecciones y reclamos que realizan a la escuela, distintos aspectos que hacen a la vida escolar. Sus reclamos se vinculan con, fundamentalmente, mejorar las condiciones en que se desenvuelven los aprendizajes. Así, creemos que es central ahondar sobre algunas ideas que los jóvenes tienen de la escolarización en ambas áreas, tal como hacemos a continuación.

\section{Las defensas y apuestas por la escolarización de los jóvenes y las diferencias con sus docentes}

Tal como decíamos en la introducción, se trata de estudiar de manera comparativa las dinámicas cotidianas de los dispositivos pedagógicos atendiendo a las prácticas de los sujetos en dos localidades en las que la pobreza urbana de las comunidades fue profundizándose. La hipótesis es que así como esas condiciones y situaciones de vida de los sujetos van cambiando y mutando conjuntamente con los procesos de transformación social y económica del país, también esas condiciones se expresan, actúan e intervienen en la cotidianeidad escolar interpelando a esos dispositivos pedagógicos. Entonces, en primer lugar nos interesa indagar qué cosas suceden efectivamente en la escolaridad de los jóvenes estudiantes en la actualidad, tal como se presenta en la Tabla $\mathrm{N}^{\mathrm{o}} 2$.

Luego de sucesivas reformas educativas formales ${ }^{6}$ y de aquello que se expresa en la cotidianeidad escolar frente a las condiciones de vida que presentan los estudiantes, lo que mayormente sucede tanto en Santa Cruz como en San Martín para los estudiantes, a partir de la Tabla $N^{0} 2$, es que se dan tareas para la casa $(80.3 \%$ y $79.6 \%$, respectivamente), utilizan libros $(83.8 \%$ y 89.4

6 Nos referimos a las reformas educativas a través de cambios estructurales en las leyes tales como la Ley Federal de Educación No 24.195 de 1993 y la Ley de Educación Nacional No 26.206 de 2006. Estas leyes cambian sustancialmente la forma de regular y concebir la educación, los saberes, los educadores y los educandos en el país. Para ver un desarrollo de aquello que producen estas reformar revisar Grinberg (2008). 
$\%$, respectivamente) y trabajan en grupos ( $83.4 \%$ y $89,9 \%$, respectivamente). La diferencia entre ambas áreas, es que para los estudiantes de San Martín, hay muchas otras cosas que suceden tal como investigar en internet $(79.4 \%)$ y debatir los temas entre todo el curso $(76.5 \%)$ con tanta fuerza como aquellas que nombramos en primer lugar. No sucede así con los estudiantes de Santa Cruz.

\section{Tabla $\mathbf{N}^{0} 2$ \\ Las actividades que se realizan en la escuela según jóvenes estudiantes de Caleta Olivia y de San Martín. En \%}

\begin{tabular}{|l|c|c|}
\hline & $\begin{array}{c}\text { Caleta Olivia } \\
(\mathbf{N}=\mathbf{2 7 9 0})\end{array}$ & $\begin{array}{c}\text { San Martín } \\
\text { (N= 1179) }\end{array}$ \\
\hline Dan tareas para la casa & 80,3 & 79,6 \\
\hline Se utilizan libros en clase & 83,8 & 89,4 \\
\hline Dan actividades para ir a la biblioteca & 36,3 & 43,5 \\
\hline Trabajan con el diario & 39,9 & 45,5 \\
\hline Dan documentales o películas & 46,5 & 53,8 \\
\hline Se usa el laboratorio & 33,2 & 29,3 \\
\hline Se usa la sala de informática & 32,8 & 43,1 \\
\hline Dan actividades para hacer en la computadora. & 44,3 & 63,9 \\
\hline Piden investigar en Internet & 51,1 & 79,4 \\
\hline Se trabaja en grupos & 83,4 & 89,9 \\
\hline Se debaten los temas entre todo el curso & 59,6 & 76,5 \\
\hline Ponen falta si llegas tarde & 58,5 & 78,6 \\
\hline Preguntan por qué llegamos tarde o por qué faltamos & 66,3 & 70,9 \\
\hline Hacen recomendaciones sobre la higiene personal & 55,1 & 45,2 \\
\hline Nos dejan llevar celular & 22,9 & 21,8 \\
\hline Sancionan si no hago la tarea. & 31,4 & 32,3 \\
\hline
\end{tabular}

Fuente y elaboración: Autor.

Pero el gran común para todos los jóvenes es que la enseñanza directa y explícita es la gran ausente. Directamente, no aparece. Enseñar no parecería ser importante a la hora de pensar la conformación del dispositivo pedagógico en la actualidad. De hecho, podríamos afirmar que una de las consecuencias que contrajeron esas reformas educativas no sólo fue la desatención en 
la enseñanza sino también en dejar en soledad ${ }^{7}$ a los docentes, porque en su gran mayoría ellos planifican solos. No hay posibilidad de pensar en formas de organización institucional ni tampoco decisiones de las autoridades para hacerlo conjuntamente. Ello es el rumbo que han marcado las reformas educativas y que se expresa en las actividades cotidianas que desarrollan las escuelas. Son docentes que dan clases a la deriva, librados a su propia suerte (Grinberg, 2008), que están solos y que se les hace muy difícil hacer y sostener sus clases ya sea porque tienen problemas personales, porque las condiciones laborales son muy precarias y/o porque no hay espacios institucionales o son insuficientes ante situaciones conflictivas que se les presentan.

Las condiciones actuales muestran que las escuelas, los estudiantes y los docentes están cada vez más solos y librados a sí mismos, las escuelas parecieran ser islas autónomas, "el sistema escolar va pareciéndose en forma creciente a una galaxia de instituciones cada vez más autónomas, cada una con su inercia. Desde el centro sólo se adjudica personal y se pagan sueldos" (Tenti Fanfani, 2011, p. 64). De lo que se trata ahora es de ser abierto, de adaptarse, de ser flexibles porque son directores, docentes y estudiantes libres y librados a su suerte, que están obligados cada vez más a hacerse a sí mismos. Por tanto, las dinámicas cotidianas de las escuelas no están signadas por la falta de rumbo sino que, justamente, el rumbo de esas dinámicas tiene que ver con los procesos de desorganización y desorientación (Langer, 2013) producto de ese "hazte a ti mismo" que, muchas veces, parece imperar en el desarrollo de esa escolaridad obligatoria.

En este sentido, sobre estos rumbos que desorientan y que van conformando las dinámicas escolares localizamos las discusiones y los debates sobre las disciplinas en las escuelas. Las disciplinas son creadoras de aparatos de saber (Foucault, 2006), de saberes y de campos múltiples de conocimiento, y en consecuencia portan discursos que serán el de la regla o la norma. La disciplina implica pensar, entre otras cosas, en la distribución de los individuos en el espacio para tener un estricto control de las presencias y las ausencias, también se sucede en las actividades y en los tiempos que deben permitir el desarrollo del proceso de enseñanza-aprendizaje, entre muchas otras cosas. Pero estas instituciones disciplinarias, entre ellas la escuela, se reconfiguran en la actualidad y adoptan nuevas características. Las reformas establecen nuevos parámetros y a

7 Así como podríamos afirmar que las instituciones y sus autoridades están en soledad para gestionar sus propios recursos, tal como dijimos en la primera dimensión de análisis. 
su vez los docentes y estudiantes también en función de esas nuevas legalidades que se van produciendo. ¿Cómo consideran jóvenes y adultos, estudiantes y docentes, el régimen disciplinario en la actualidad? ¿Cuáles son las distinciones que surgen entre ellos? ¿Qué nos están diciendo estas diferencias? La Tabla $N^{\mathrm{o}} 3$ nos ayuda a responder algunas de estas preguntas.

Tabla $\mathbf{N}^{0} 3$

\section{Las ideas de estudiantes y docentes de Caleta Olivia y de San Martín acerca de la disciplina. En \%}

\begin{tabular}{|l|c|c|c|c|}
\hline \multirow{2}{*}{} & \multicolumn{2}{|c|}{ Caleta Olivia } & \multicolumn{2}{c|}{ San Martín } \\
\cline { 2 - 5 } & $\begin{array}{c}\text { Docentes } \\
(\mathbf{N = 1 1 7 )}\end{array}$ & $\begin{array}{c}\text { Estudiantes } \\
(\mathbf{N}=\mathbf{2 7 9 0})\end{array}$ & $\begin{array}{c}\text { Docentes } \\
(\mathbf{N}=\mathbf{6 3})\end{array}$ & $\begin{array}{c}\text { Estudiantes } \\
(\mathbf{N = 1 1 7 9})\end{array}$ \\
\hline Muy rígida & 2,6 & 19,1 & 0 & 9,2 \\
\hline Rígida & 4,3 & 20,5 & 11,1 & 17,5 \\
\hline Flexible & 22,2 & 14,1 & 34,9 & 13,9 \\
\hline Muy flexible & 17,1 & 7,7 & 23,8 & 6,0 \\
\hline Ni tan rígida, ni tan flexible & 43,6 & 27,2 & 20,6 & 43,7 \\
\hline
\end{tabular}

Fuente y elaboración: Autor.

La primera y principal lectura que surge de la Tabla $\mathrm{N}^{0} 3$ es que hay tensiones considerables entre las prácticas discursivas de los jóvenes por un lado y las de los adultos por el otro, en ambas áreas: a) Hay porcentajes considerablemente más significativos de los jóvenes para las respuestas que hacen referencia al régimen disciplinario como rígido y muy rígido en comparación a los adultos. b) Los porcentajes ascienden lentamente de estudiantes hacia docentes cuando la respuesta es régimen disciplinario flexible. c) Los porcentajes son considerablemente más significativos de las respuestas de docentes acerca de un régimen disciplinario demasiado flexible con respecto a las respuestas de estudiantes. Sucede una distinción para ambas áreas que tiene que ver con la respuesta "ni tan rígida, ni tan flexible". Los jóvenes en San Martín parecerían darse cuenta en mayor medida que sus pares de Santa Cruz de los vaivenes y sinsentidos que, muchas veces, las normativas tienen en la producción de la escolaridad. A su vez, al revés sucede con los docentes. Son en Santa $\mathrm{Cruz}^{8}$ quienes en mayor medida que sus pares de

8 Vale aclarar que durante los años de toma de encuestas en Santa Cruz hubieron fuertes conflictos y luchas docentes en función de las condiciones salariales. 
San Martín dan cuenta de esta situación de fragilidad, inestabilidad e incertidumbre en el sistema educativo en relación a las normas y a su puesta en funcionamiento.

Sin embargo, paradójicamente, los jóvenes y los adultos en contexto de pobreza urbana esperan muchas cosas de la escuela. En la Tabla No 4 observamos sus ideas acerca de la escuela en relación al trabajo, al futuro, al acceso a estudios posteriores, a la ciudadanía y a las defensas de sus derechos, entre otras cosas.

\section{Tabla $\mathbf{N}^{\circ} 4$}

\section{La importancia de la escuela para jóvenes estudiantes y docentes de Caleta Olivia y de San Martín. En \%}

\begin{tabular}{|l|c|c|c|c|}
\hline & \multicolumn{2}{|c|}{ Caleta Olivia } & \multicolumn{2}{c|}{ San Martín } \\
\cline { 2 - 5 } & $\begin{array}{c}\text { Docentes } \\
(\mathbf{N = 1 1 7})\end{array}$ & $\begin{array}{c}\text { Estudiantes } \\
(\mathbf{N = 2 7 9 0 )}\end{array}$ & $\begin{array}{c}\text { Docentes } \\
(\mathbf{N = 6 3 )}\end{array}$ & $\begin{array}{c}\text { Estudiantes } \\
\text { (N=1179) }\end{array}$ \\
\hline Sirve para el trabajo & 47 & 74,0 & 84,1 & 93,0 \\
\hline $\begin{array}{l}\text { Sirve para continuar } \\
\text { estudiando }\end{array}$ & 70,9 & 52,0 & 74,6 & 76,8 \\
\hline $\begin{array}{l}\text { Forma para ser buen } \\
\text { ciudadano }\end{array}$ & 60,7 & 33,0 & 84,1 & 73,5 \\
\hline $\begin{array}{l}\text { Forma para ser buena } \\
\text { persona }\end{array}$ & 56,4 & 35,7 & 73 & 64,2 \\
\hline $\begin{array}{l}\text { Aunque sea poco, } \\
\text { algo pueden hacer }\end{array}$ & 39,3 & 21,2 & 60,3 & 39,6 \\
\hline $\begin{array}{l}\text { No es importante } \\
\text { lo que enseña la escuela }\end{array}$ & 0,9 & 2,5 & 1,6 & 7,4 \\
\hline
\end{tabular}

Fuente y elaboración: Autor.

De esta tabla, nos damos cuenta que los jóvenes esperan en mayor proporción que los adultos que la escuela los forme para el trabajo, tanto en Santa Cruz como en San Martín. A la inversa, los docentes esperan en mayor proporción que los estudiantes que la escuela forme para ser buenos ciudadanos y buenas personas, en ambas áreas. En cambio, suceden tendencias diferentes en cuanto a la justificación de que sirve para continuar estudios posteriores. En Santa Cruz, en mayor medida son los docentes quienes dicen eso y en San Martín, son los estudiantes. 
Más allá de esas especificidades, con esta tabla desmontamos frases que se escuchan y producen en la escuela y en la sociedad sobre el no interés, ganas y gustos de los jóvenes. Cuando se empieza a indagar un poco más, ello no es lo que predomina. El "no hay mucho interés en la escuela", el que muchos chicos no van, o que la escuela "no sirve absolutamente para nada" no son expresiones que representen la sensación generalizada de la experiencia escolar por parte de los estudiantes en escuelas en contextos de pobreza urbana, tanto de Santa Cruz como de San Martín. La asistencia a la escuela, aunque muchas veces discontinua, más que la pérdida de sentido de la escuela para los jóvenes en contextos de pobreza urbana "evidencia una importante significación en las historias de vida relevadas, no necesariamente asociada a visiones románticas sobre su realidad ni a posiciones optimistas, sino más bien a una búsqueda de un espacio y un tiempo que los inscriba" (Redondo, 2004, p. 129). Justamente, por ello, a continuación mostramos en la Tabla $N^{0} 5$ las razones que jóvenes estudiantes y docentes de ambos lugares dan de la asistencia a la escuela.

Tabla $\mathbf{N}^{0} 5$

Las razones de asistencia a la escuela para docentes y estudiantes de Caleta Olivia y de San Martín. En \%

\begin{tabular}{|l|c|c|c|c|}
\hline \multirow{2}{*}{} & \multicolumn{2}{|c|}{ Caleta Olivia } & \multicolumn{2}{c|}{ San Martín } \\
\cline { 2 - 5 } & $\begin{array}{c}\text { Docentes } \\
(\mathbf{N = 1 1 7 )}\end{array}$ & $\begin{array}{c}\text { Estudiantes } \\
(\mathbf{N = 2 7 9 0 )}\end{array}$ & $\begin{array}{c}\text { Docentes } \\
(\mathbf{N = 6 3 )}\end{array}$ & $\begin{array}{c}\text { Estudiantes } \\
\text { (N=1179) }\end{array}$ \\
\hline Queda cerca de la casa & 75,2 & 47,5 & 58,7 & 53,9 \\
\hline Los hermanos concurre/n también & 75,2 & 30,5 & 52,4 & 36,2 \\
\hline $\begin{array}{l}\text { Hay un buen ambiente socialy } \\
\text { cultural }\end{array}$ & 30,8 & 27,1 & 33,3 & 57,9 \\
\hline No hay chicos con problemas & 2,6 & 6,1 & 11,1 & 24,2 \\
\hline $\begin{array}{l}\text { Es prestigiosa/ recomendada/ } \\
\text { reconocida }\end{array}$ & 25,6 & 17,6 & 27 & 46,5 \\
\hline No había lugar en otras escuelas & 13,7 & 12,1 & 11,1 & 17,0 \\
\hline
\end{tabular}

Fuente y elaboración: Autor.

Acá hay fuertes diferencias entre adultos y jóvenes en las escuelas tanto de Santa Cruz como de San Martín, aunque con tendencias opuestas en 
algunos casos. Para docentes de ambas áreas, en mayor medida las razones principales de asistencia a la escuela de los estudiantes tienen que ver con la cercanía así como con que sus hermanos concurren allí. En cambio para los estudiantes que concurren a escuelas en San Martín, la razón principal es que haya buen ambiente social y cultural $(57,9 \%)$ y para los estudiantes que concurren a escuelas en Santa Cruz, si bien es que les queda cerca (47,5\%) ello aparece más matizado y con menos fuerza porque no hay tanta distancia entre ese porcentaje y el resto de las justificaciones, como también sucede con los estudiantes en San Martín. Es significativo el porcentaje que aparece en los estudiantes de San Martín que dicen que asisten allí porque es prestigiosa, recomendada y reconocida con el $46.5 \%$. Con ello se podría pensar que los jóvenes en contextos de pobreza urbana si bien no eligen en un primer momento la escuela a la que van, cuando ya están allí sí lo hacen por aquello que la escuela es y por aquello que allí viven. Ellos apuestan, desde formas diversas y con diferentes maneras de expresión, por su escuela. No asisten porque sí y tienen sus razones de por qué es importante concurrir ya que les va a servir de alguna u otra forma para sus vidas.

En definitiva, los jóvenes quieren ir a la escuela. En el caso de las escuelas emplazadas en contextos de pobreza urbana la valorizan, la eligen cuando están allí y, por tanto, la defienden porque los ayuda a defender sus derechos. Entonces, si bien las dinámicas que asume la trama escolar y las características que están presentando los dispositivos pedagógicos en estas sociedades, profundizando los procesos de fragmentación educativa del nivel secundario, se generan prácticas de defensa a la escuela y a la educación por parte de los jóvenes en ambos lugares. No sólo quieren a la escuela que van sino que no se quieren cambiar de ella, tal como se muestra a continuación.

Tabla N ${ }^{0} 6$

Jóvenes estudiantes de Caleta Olivia y de San Martín que les gustaría o que no les gustaría cambiarse de escuela. En \%

\begin{tabular}{|l|c|c|}
\hline & $\begin{array}{c}\text { Caleta Olivia (N= } \\
\mathbf{2 7 9 0}\end{array}$ & $\begin{array}{c}\text { San Martín } \\
\text { (N= 1179) }\end{array}$ \\
\hline Le gustaría cambiar de escuela & 24,5 & 13,5 \\
\hline No le gustaría cambiar de escuela & 70,8 & 83,6 \\
\hline
\end{tabular}

Fuente y elaboración: Autor. 
Al $83 \%$ de los estudiantes de San Martín y al 70\% de los estudiantes de Santa Cruz no les gustaría cambiar de escuela y al 13\% y 24\% respectivamente de ellos sí. Es decir, a 8 de cada 10 jóvenes en San Martín y 7 de cada 10 en Santa Cruz les gustaría permanecer en la escuela en la que están. Esto es un dato central que indica que algo de todo el orden que sucede dentro de las escuelas los "convence" para seguir estando allí. Entonces, sabiendo que una gran mayoría de estudiantes no quieren cambiarse de escuela, damos sentido a las razones que los estudiantes expresan de sus defensas en el cotidiano escolar. En general, nos encontramos que quieren quedarse en la escuela porque están cómodos y les gusta. También, otra fundamentación fue el nivel o tipo de educación que se brinda, aunque en menor medida para ambas áreas. Los estudiantes en contextos de pobreza urbana se quieren quedar en la escuela -además de porque están cómodos y más allá de "por los amigos", que también es algo que dicen los jóvenes indistintamente, por la "cercanía de la casa" y el "buen ambiente". Estas defensas de sus escuelas, expresan algunas de las luchas sociales por la escolarización a la que nos referimos. Pero no solamente. Esas luchas son producto y consecuencia de las que expresan los padres y las madres de los estudiantes tanto fuera como dentro de las escuelas, tal como desarrollamos a continuación.

\section{"Remarla" para sostener la educación de los jóvenes}

Las luchas que realizan los jóvenes se asemejan y corresponden a aquellas que realizan sus familias en contextos de pobreza urbana de ambas áreas, producen cambios en las dinámicas cotidianas de los dispositivos pedagógicos e involucran las pugnas por estar, permanecer y tener mejor escuela. Tal como hemos sugerido ya, las prácticas de los jóvenes estudiantes en escuelas secundarias en contextos de pobreza urbana tienen sus motivos y sus causas, tal como nos decían algunos estudiantes: "depende la clase de vida" o "siempre hay un motivo" (Grinberg y Langer, 2012; Langer, 2013). Aquí específicamente, se propone que las prácticas que despliegan tanto los jóvenes como sus familias en estos contextos han tendido y tienden a resignificar la regulación de los dispositivos tal como se producen en las propuestas oficiales. Son las situaciones vividas y las prácticas que realizan los sujetos en la vida diaria para sobrevivir que se dirimen cotidianamente en 
el desarrollo de la escolaridad. Proponemos que la escuela oficia, de muy diversas maneras, como lugar de resistencia y afirmación tanto para los jóvenes como para sus familias. Así, aquí nos concentramos en las luchas que realizan por la escolarización y por sostener la educación cotidianamente a través de las entrevistas en profundidad realizadas.

En los barrios periféricos de ambas áreas nos encontramos con mujeres y hombres que quieren mejorar las condiciones de vida de sus hijos y las de sus familias. Son familias que buscan, hacen, desafían, sostienen, insisten, piden por sus derechos, piden "ganar derechos". Nos referimos a un conjunto de acciones de la población liminar (Foucault, 2007) que involucran tanto la resistencia hacia las consecuencias del capitalismo como a las formas de disciplinamiento y control. Todas estas acciones las pensamos desde la noción de "luchas" que les permiten, como nos decía una de ellas que es referente en una organización social de uno de los barrios más desiguales de San Martín:

Todas somos mujeres luchadoras realmente, que venimos haciendo. Somos mujeres luchadoras para poder dar el ejemplo a las chicas jóvenes, que puedan ayudarse entre ellas. Siempre hay alguno que quiere bajarse. No. Hay que seguir luchando y poder lograr lo que uno quiere hacer. Y unirse (Registro de entrevista a mujer, José León Suárez, Agosto de 2015).

En el discurso de lucha, se suceden las afirmaciones, las posibilidades de ayudar, poder lograr aquello que se quiere y anhela, las solidaridades colectivas. Algo que aparece también con mucha fuerza en las entrevistas en profundidad tanto de los jóvenes como de sus familias es la posibilidad de mejorarlos, describieron cosas que cambiarían del barrio en el que viven. Es significativo que muy pocos nos dijeran que cambiarían de barrio. Nos encontramos con respuestas que hacen al mejoramiento de los mismos y no al irse. Como referenciamos más arriba, porque quieren algo mejor para ellos, para sus familias y para sus hijos. Ello, creemos, implica quedarse en sus lugares, permanecer, luchar por esos espacios para que estén mejor. Las principales respuestas tuvieron que ver con asfaltar las calles de tierra, la limpieza y la iluminación del barrio. Por otra parte, muchos sostuvieron la necesidad de que haya más árboles, espacios verdes y parques para la recreación. Algunos se refirieron a "las necesidades para mejorar la calidad de vida". Este discurso de mejora, se sucede centralmente sobre las escuelas a las que concurren.

Estas distintas formas de vida y de destinos que se construyen en este contexto expresan los lugares que adquieren las luchas para pensar un fu- 
turo distinto. El escenario en el cual luchan para contrarrestar las desigualdades de los proyectos de vida, viene asociado a redefinir un destino que se presenta como inmutable. Así, nos encontramos con sujetos que la pelean: hombres jefes de familia que están desocupados y salen a hacer changas, mujeres jefes de familia que están solas y salen adelante, familias que están constituidas en su totalidad y aún les cuesta un montón porque no hay trabajo, no hay estabilidad. En esas peleas, refuerzan el "yo", el "soy" y el "tengo". Yo, soy y tengo en la lógica de las sociedades de gerenciamiento (Grinberg, 2008) significa la configuración de un sujeto que busca, que se adapta, realiza los cambios necesarios en el marco del mundo flexible, "en el presente es llamado a realizarse, a crear y crearse en su propio arte de vivir" (Grinberg, 2008, p. 200). Esta lógica excluye, extingue a aquellos que "no se adaptan", "no cambian", "no aprenden a aprender", afecta a aquellos que no se adaptan a las condiciones de enseñanza y aprendizaje; el individuo desde esta lógica será responsable individual de su fracaso por sostener su educación. No importan las condiciones de existencia, la desigualdad ni ninguna variable estructural porque aprender a aprender se volvió el eje de la sociedad. Y, quienes resultan excluidos, difícilmente podrán considerarse competitivos en el futuro y quedarán marginados de toda sociedad. He aquí a los sujetos en los barrios que nos referimos. Por un lado son quienes quedan fuera, pero por el otro son quienes la pelean, se adaptan, cambian, hacen de todo, "tienen ocho manos" tal como nos decía un grupo de jóvenes en la escuela. Nos referimos a mujeres malabaristas que sostienen con su trabajo el bienestar económico y afectivos de sus hogares (Faur, 2014).

Especialmente, las mujeres de los barrios priorizan su familia y a sus hijos en vez de su vida propia. Dejan todo por ello. Salen adelante. Luchan contra todo para que sus hijos estén bien. Hacen de todo. Son mujeres, madres, que se esfuerzan por cambiar las condiciones existentes y expresan su disconformidad por las formas de vida. Las luchas de las madres que se observan cotidianamente en las escuelas en comunidades en condición de pobreza están estrechamente en vinculación con estas historias de postergación de deseos, sueños, dolores, sufrimientos, de abuso de autoridad, de relegación, de creencias, tal como nos cuenta una mujer en San Martín:

Yo no tengo problemas, no le busco problemas a la vida directamente. La vivo día a día, ya está. Los problemas míos los deje atrás, en el pasado. Ya fue. Me río [Se ríe y parece nerviosa]. Yo me rio y le pongo ganas a la vida. Ya no me amargo por nada. Viví muchas cosas feas en mi vida. Estaba pre- 
sa, mi problema era salir. No podía salir. Tenía mi hermano preso, tenía problema de que salga mi hermano. Cuando falleció mi hermano, ya está. Creo que fue mi gran problema y [se emociona y llora] todavía la sigo adelante. No tengo problema. Mi problema es que mis hijos no pasen lo que yo pase. No vivan lo que yo pase. No vivan lo que yo vi. Y sacarlos adelante a ellos. Reírme de la vida y no buscarle la quinta pata al gato. Ya está. Lo vivido está vivido. Buscando la alegría de ellos. No la tristeza. Qué se yo. Creo que la tristeza me la dejo para mí a la noche. Mostrarle a ellos que la vida es linda [se le caen más lágrimas y se las seca]. Que hay cosas lindas para vivir. Que no busquen lo malo. Porque en lo malo está lo feo (Registro de entrevista a mujer, José León Suárez, junio de 2015).

Son mujeres con diversos y múltiples problemas pero que los dejan al costado para ponerle ganas a la vida, para que sus hijos no pasen lo mismo que ellas. El problema de esta mujer es que sus hijos no pasen lo mismo que ella, sacarlos adelante. Apostando por la alegría, la risa, las ganas, los deseos, lo lindo de la vida. No son sólo mujeres que han sido despojadas de sus derechos y sueños, sino que son quienes han luchado y luchan por volver a recuperarlos. Tal como nos decía otra madre, "si uno quiere, puede":

Yo he trabajado, he estudiado, he trabajado en cinco trabajos, crio mis hijos, mantengo mi casa. Si uno quiere puede (Registro de entrevista a madre de estudiante, Caleta Olivia, Septiembre 2014).

Las historias con que nos encontramos dan cuenta de la no resignación a las situaciones de vida que conduce las condiciones de privación material, a la no aceptación de un destino ineluctable. Son historias de esfuerzo, de pelearla, de trabajar y de cuidar para "tener un lugar" y transmitir la experiencia de vida a sus hijos, asumiendo posturas sacrificadas ante condiciones de vida adversas para que como nos decía otra madre sepan "lo que es valorar, lo que es trabajar, lo que es llevar un plato a la mesa, lo que es comprar así sea una visera". O tal como nos lo decía otra madre, "de remarla":

Yo toda la vida estuve sola con mis hijos, y sola los tuve así también. Soy yo sostén de familia. Hace diez años he estado sin trabajo, pero la he remado. Y como todo, si uno quiere sale adelante y puede (Registro de entrevista a mujer, José León Suárez, Mayo de 2014).

Son mujeres que están solas con sus hijos. Son sostén de familia. Sostienen a sus familias con o sin trabajo y como dice Butler "no puede haber per- 
sistencia en la vida sin, al menos, algunas condiciones que hagan 'vivible' una vida" (Butler, 2009, p. 40). La posibilidad de ser sostenidos y sostener se apoyan en esas condiciones de vida que remarca esta mujer: ser sola, ser sostén, estar sin trabajo. Vuelve a aparecer en este discurso el "si uno quiere, puede". Si uno quiere, sale adelante. Hay una creencia generalizada que el éxito o el fracaso, la posibilidad de que vaya bien o no una vida, es meramente una responsabilidad personal y no social. Como sostuvimos al principio del recorrido, la idea de comunidad se fortalece en este contexto, porque los sujetos adquieren responsabilidad para gestionarse a sí mismos con los recursos escasos con los que cuentan para sostener su vida, la de los otros, para sostener sus derechos, entre ellos como ya vimos, la escolaridad. También, se fortalece la posibilidad de resistir, de ser, hacer y tener. Es en esta encrucijada y contradicción que pensamos las luchas sociales por la escolarización, desde las contradicciones desde las cuales se desarrolla la escolaridad en estos barrios. Es decir, entre las imposibilidades de las políticas que marcan rumbos que desorganizan y las posibilidades que van expresando los sujetos en aquellos barrios en el que las desigualdades se fueron profundizando, tal como se puede ver en el relato de la siguiente mujer acerca de cómo "todo está cambiado" y por ello su apuesta a la educación de ella y de sus hijos:

Hoy en día les podemos decir a nuestros hijos que estudien. Que es lo mejor que se puede hacer. Nos damos cuenta que todo está cambiado. No es lo mismo de antes. Nosotros tenemos que estudiar para que nuestros hijos sigan el ejemplo de lo que estamos haciendo hoy en día. No de lo que hicimos y de los errores que tuvimos. De lo bueno que estamos sacando ahora. Hoy en día por la edad que tengo puedo decir `quiero terminar mi secundario ` para prevenirme el día de mañana. Para poder ir a pedir un trabajo. Para poder decirle a mis hijos estudien, estudien porque del estudio se saca lo bueno. No lo malo que se ve en las calles (Registro de entrevista a madre, Caleta Olivia, marzo, 2015).

Dar el ejemplo con el estudio, prevenirme el día de mañana, poder pedir un trabajo, poder decirle a sus hijos que estudien son los rasgos que a través de esta mujer expresan las luchas sociales por la escolarización. Muchos de los jóvenes con los que nos encontramos en estos barrios tienen este discurso y presentan sus luchas de esta forma. Ya no son los jóvenes de hace algunas décadas que querían dejar la institución escolar. Son jóvenes y familias que pugnan por un lugar en la institución, por un lugar en el mundo. 
Claro que aquí las luchas son pensadas como prácticas en plural porque nos referimos a sujetos que despliegan una cantidad de acciones que pueden ser diversas y hasta contradictorias, y que ponen en juego y cuestionan las condiciones en que se desenvuelve en el presente con vistas a las repercusiones no solo a corto plazo sino, principalmente, a largo plazo. De hecho, no todas las luchas son iguales, no hay una resistencia per se. Pero ellas tienen en común que intentan superar las condiciones en las que viven. Son prácticas con el objeto de construir una mejor existencia, como decíamos antes "estar mejor", aunque no de modos homogéneos. Enfrentan y luchan de diferentes formas contra condiciones intolerables, sea en Santa Cruz o en San Martín, aunque con matices distintos que tienen que ver con las especificidades que fuimos recorriendo al principio de este texto.

\section{Reflexiones finales}

En este texto quisimos encontrar dimensiones comparativas de dos contextos alejados físicamente pero cercanos por las similitudes de algunas de las consecuencias sufridas en sus territorios producto de las crisis sociales de los últimos cuarenta años. Así, pobreza, escolarización y luchas fueron las nociones que recorrimos para explorar esas diferencias y semejanzas.

$\mathrm{Al}$ principio del recorrido, encontramos que los flujos poblacionales hacia estas áreas son centrales para entender la profundización de las desigualdades sociales hacia la actualidad. Allí, observamos que es una población que no se quiere volver a ir de esos lugares a los que llegaron. Sino que se quieren quedar, quieren mejorarlos y cambiarlos. Sobre todo porque las condiciones y situaciones de vida de los jóvenes y las de sus familias se han ido pauperizando durante las últimas décadas. Ello lo mostramos a través de cómo los jóvenes que estudian a la vez trabajan, viven en hogares sostenidos por planes o ayudas sociales y en los que los jefes de hogares, muchas veces, no tienen la escolaridad mínima.

A partir de allí, trabajamos las valoraciones de jóvenes estudiantes y de docentes de Santa Cruz y de San Martín sobre la escuela, cuál es su importancia, por qué asisten, lo que sucede allí adentro, cómo es la disciplina, qué hacen y si les gustaría cambiarse de escuela o no. Mostramos, que no hay tantas diferencias entre ambas áreas como sí las hay entre las generaciones, entre los jóvenes estudiantes y los adultos docentes. Así, una generación, 
coincidimos con Vommaro (2014), "puede ser comprendida a partir de la identificación de un conjunto de sujetos que comparten la experiencia de lo que perciben como un conflicto, un problema. Así, el vínculo generacional aparece y se constituye como efecto de un proceso de subjetivación, ligado con una vivencia común en torno a una experiencia de ruptura" (p. 23). Entre jóvenes y adultos hay diferencias y rupturas de cómo sienten a la escuela, para qué sirve, por que asisten y cómo ven a la disciplina en la actualidad. Hubieron pequeñas diferencias entre los territorios que tuvieron que ver con, por ejemplo, los conflictos docentes, las tomas estudiantiles y, también, los problemas barriales.

Por último, recorrimos algunas de las valoraciones de las familias de los jóvenes para entender esas luchas como defensas, apuestas y peleas por tener un lugar en una sociedad, como dijimos, con déficit de lugares ocupables (Castel, 1996). Estas insistencias de las madres, centralmente, para que sus hijos estén en la escuela, alejarlos de la calle y las drogas, sostenemos aquí pueden contribuir a entender y caracterizar los dispositivos escolares en el presente en ambos territorios así como también a las luchas de los jóvenes por su escolarización tanto como sus prácticas y expresiones políticas actuales.

Entonces, las pugnas y luchas cotidianas de los jóvenes por sostener la educación y por apostar a la escuela tienen que ver con, por un lado, las dificultades que tienen y viven en condiciones de pobreza para sobrevivir todos los días tal como trabajamos en los primeros apartados, y por otro lado en sus ideas, valoraciones y sentires sobre la importancia de la educación y de la escuela en un mundo, que como nos dijeron, está cambiado.

Nos encontramos a lo largo del texto con relatos y valoraciones en relación a la educación porque descansa en el porvenir y, entonces, podemos pensar a las luchas por la escolarización en relación con esa esperanza hacia adelante. Los jóvenes en ambos lugares de la investigación creen en la escuela como espacio de formación, socialización, donde están seguros y cómodos, que pueden y quieren estar. Así, la escuela, en algunos casos, se conforma como lugar de pertenencia y decisión aún cuando las condiciones no son las mejores desde las visiones que recuperamos de los jóvenes.

En definitiva, nos referimos a luchas que demandan y piden por más y mejor escolaridad, a través de reclamos para "estar bien preparados", denuncias de los jóvenes porque se ven asimismo "cada vez más burros", porque ven que sus docentes no tienen buenas condiciones laborales, para "po- 
der hacer lo que uno quiere", etc. Estas acciones, entre muchas otras, son formas que adquieren las luchas para estar mejor en las escuelas, para mejorar la vida. En ese marco, los jóvenes en condición de pobreza producen nuevas formas de interactuar con las instituciones, consigo mismos y, también, con el Estado.

Estudiamos esas luchas como prácticas cotidianas de los jóvenes, no siempre explícitamente formuladas y/o directas, que les permiten expresar sus palabras políticas ante situaciones que no los convencen o no les gustan y en función del malestar que viven en sus barrios. Nos referimos a prácticas de negociación y de rechazo más que de transformación social, a prácticas impulsivas o no intencionales que emergen del cuerpo y, también, a acciones que pueden ser resultado de decisiones planteadas desde el humor, los deseos, las ganas y las apuestas por esas promesas de formación futuras que la escuela sigue siendo hoy aún para los jóvenes que viven en condición de pobreza. El estudio de estas prácticas nos permite comprender el protagonismo de los jóvenes, entendido aquí como aquellas acciones que ponen en juego todos los días dentro y fuera de las escuelas.

\section{Bibliografía}

Appadurai, Arjun (2007). El rechazo de las minorías. Ensayo sobre la geografía de la furia. Barcelona: Ensayo Tus Quets editores.

Butler, Judith (2009). Marcos de guerra. Las vidas lloradas. Buenos Aires: Paidós. Castel, Robert (1996). La metamorfosis de la cuestión social. Una crónica del salariado. Buenos Aires: Paidós.

Davis, Mike (2007). Planeta de ciudades miseria. Madrid: Foca Ediciones.

De Marinis, Pablo (2002). Ciudad, 'cuestión criminal' y gobierno de poblaciones. Política y Sociedad. Revista de la Facultad de Ciencias Políticas y Sociología de la Universidad Complutense de Madrid, 39(2), 319-338.

Faur, Elena (2014). El cuidado infantil en el siglo XXI. Mujeres malabaristas en una sociedad desigual. Buenos Aires: Siglo XXI editores.

Fistetti, Francesco (2004). Comunidad. Léxico de política. Buenos Aires: Nueva Visión.

Foucault, Michel (2006). Seguridad, territorio, población. Buenos Aires: FCE. (2007). Nacimiento de la biopolítica. Buenos Aires: FCE. (2007a). La arqueología del saber. Buenos Aires: Siglo Veintiuno editores. 
Grinberg, Silvia (2006). Gubernamentalidad, vida escolar y violencia en emplazamientos urbanos marginales. Claroscuro, Revista del Centro de Estudios sobre Diversidad Cultural, V(5), 110-124.

(2008). Educación y poder en el siglo XXI. Gubernamentalidad y pedagogía en las sociedades de gerenciamiento. Buenos Aires: Ed. Miño y Dávila.

Grinberg, Silvia y Langer, Eduardo (2012). Education and Governmentality in Degraded Urban Territories: From the Sedimented to the Experience of the Actual. En: D. Cole (Ed.), Surviving economic crises through education (pp. 149-164). Sydney: Peter Lang Publishing.

Harvey, David (2012). Las condiciones de la posmodernidad. Buenos Aires: Amorrortu.

Hessel, Stephane (2006). Comprometeos. Ya no basta con indignarse. Conversaciones con GillesVanderpooten. Barcelona: Ediciones Destino.

Holston, James (2009). La ciudadanía insurgente en una era de periferias urbanas globales. Un estudio sobre la innovación democrática, la violencia y la justicia en Brasil. En: G. Delamata (Coord.), Movilizaciones sociales: ¿nuevas ciudadanías? Reclamos, derechos, Estado en Argentina, Bolivia y Brasil. Buenos Aires: Biblos.

Kristeva, Julia (1999). Sentido y sinsentido de la rebeldía. Literatura y psicoanálisis. Santiago de Chile: Cuarto Propio.

Langer, Eduardo (2013). Dispositivos pedagógicos en las sociedades de control: prácticas de resistencia de estudiantes y regulación de las conductas en escuelas secundarias en contextos de pobreza urbana del Partido de San Martín. (Tesis doctoral de la Universidad de Buenos Aires). Buenos Aires: Facultad de Filosofía y Letras (UBA).

Langer, Eduardo, Venturini, María Eugenia y Grinberg, Silvia (2013). Abordaje metodológico. En: Silvia Grinberg (Coord.), La escuela not dead. Dispositivos pedagógicos, territorios y desigualdad. Santa Cruz-Argentina: Ed. UNPA.

Lefebvre, Henri (1978). El derecho a la ciudad. Barcelona: Ediciones Península.

Onfray, M. (2011). Politica del rebelde. Tratado de resistencia e insumisión. Barcelona: Anagrama/ Colección Argumentos.

Pérez, Andrés (2012). Biopolítica y territorio. Procesos de re-configuración urbana. El caso de Caleta Olivia, provincia de Santa Cruz. Espacios Nueva Serie. Estudios de Biopolitica, 1(7), 289-303.

PrévôtSchapira,Marie(2002). BuenosAiresenlosaños 90:metropolizaciónydesigualdades. Revista EURE, 28(85), 31-50. Recuperado de http://www.scielo. 
cl/scielo.php?script=sci_arttext\&pid=S0250-71612002008500003, 20 de Agosto de 2015.

Redondo, Patricia (2004). Escuelas y pobreza. Entre el desasosiego y la obstinación. Buenos Aires: Paidós.

Reguillo, Rossana (2012). Culturas juveniles. Formas políticas del desencanto. Buenos Aires: Siglo veintiuno editores.

Rose, Nikolas (1996). Identidad, genealogía, historia. En: S. Hall y P. Du Gay (Comps.), Cuestiones de identidad cultural (pp. 214-250). Madrid: Amorrortu Editores.

(1996a). ¿The death of the social? Re-figuring the territory of government. Economy and Society, 25, 327-356.

Segura, Ramiro (2006). Segregación residencial, fronteras urbanas y movilidad territorial. Un acercamiento etnográfico. Cuadernos del Ides, 9, 3-25. Recuperado de http://ides.org.ar/wp-content/uploads/2012/03/cuadernos9_Segura.pdf, 8 de Octubre de 2011.

Shammah, Cinthia (2009). El circuito informal de los residuos. Los basurales a cielo abierto. Buenos Aires: Espacio Editorial.

Silberman-Keller, Diana, Bekerman, Zvi, Giroux, Henri y Burbules, Nicholas (2011). Cultura popular y educación. Imágenes espejadas. Buenos Aires: Miño y Dávila Editores.

Tenti Fanfani, Emilio (2011). La enseñanza media hoy: masificación con exclusión social y cultural. En: G. Tiramonti y N. Montes N. (Ed.), La escuela media en debate. Problemas actuales y perspectivas desde la investigación (pp. 53-72). Buenos Aires: Manantial-FLACSO.

Vommaro, Pablo (2014). Juventudes, políticas y generaciones en América Latina: acercamientos teórico conceptuales para su abordaje. En: Sara Victoria Alvarado, y Pablo Vommaro (Comps.), En busca de las condiciones juveniles latinoamericanas. Ciudad Autónoma de Buenos Aires: Clacso; Tijuana: El Colegio de la Frontera Norte, El Colef; Manizales: Universidad de Manizales; Sabaneta: Cinde.

Wacquant, Louis (2007). Los condenados de la ciudad. Gueto, periferia y Estado. Buenos Aires: Siglo XXI.

Zibechi, Raúl (2008). Territorios en resistencia. Cartografia política de las periferias urbanas latinoamericanas. Buenos Aires: Ed. La Vaca. 